\title{
AN INEQUALITY FOR DERIVATIVES OF POLYNOMIALS WHOSE ZEROS ARE IN A HALF-PLANE
}

\author{
FARUK F. ABI-KHUZAM
}

\begin{abstract}
Let $Q$ be a real polynomial of degree $N$ all of whose zeros lie in the half-plane $\operatorname{Re} z \leqslant 0$. Let $M(r, Q)$ be the maximum of $|Q(z)|$ on $|z|=r$ and $n(r, 0)$ the counting function of the zeros of $Q$. It is shown that the inequality $M\left(r, Q^{\prime}\right) \leqslant$ $(2 r)^{-1}\{N+n(r, 0)\} M(r, Q)$ holds for $r>0$. It is also shown that Bernstein's inequality characterizes polynomials.
\end{abstract}

1. Introduction. Let $Q$ be a complex polynomial of degree $N(>0)$. For each positive real number $r$, let

$$
M(r, Q)=\max _{|z|=r}|Q(z)| .
$$

According to a result of S. Bernstein [2, p. 221]:

$$
M\left(r, Q^{\prime}\right) \leqslant(N / r) M(r, Q) \quad(r>0) .
$$

If none of the zeros of $Q$ lies in the disk $|z|<r$, then $M\left(r, Q^{\prime}\right)$ satisfies the inequality [1]:

$$
M\left(r, Q^{\prime}\right) \leqslant(N / 2 r) M(r, Q) \quad(r>0),
$$

which was conjectured by Erdös and proved by Lax.

If some, but not all, of the zeros of $Q$ lie in $|z|<r$, is there an inequality satisfied by $M\left(r, Q^{\prime}\right)$ that "interpolates" between (2) and (3)?

In order to answer this question we introduce the function $n(r, 0)=n(r, 0 ; Q)$ which is defined as follows:

For $r>0, n(r, 0)$ equals the number of zeros of $Q$ in the disk $|z| \leqslant r$, where each zero is counted as many times as its multiplicity indicates. For $r=0, n(r, 0)$ equals the multiplicity of 0 as a zero of $Q$.

The function $n(r, 0)$ is called the counting function of the zeros of $Q$. It is clearly a step function which is nondecreasing and satisfies

$$
n(r, 0) \leqslant N \quad(r \geqslant 0) .
$$

With this notation an answer to the question posed above is suggested by the following:

CONJECTURE. Let $Q$ be a polynomial of degree $N(>0)$, and let $n(r, 0)=n(r, 0 ; Q)$ be the counting function of its zeros. Then

$$
M\left(r, Q^{\prime}\right) \leqslant \frac{N+n(r, 0)}{2 r} M(r, Q) \quad(r>0) .
$$

Received by the editors January 2, 1982 and, in revised form, December 16, 1982.

1980 Mathematics Subject Classification. Primary 30C10. 
More precisely, the function $n(r, 0)$ in $(5)$ may be replaced by $n(r-, 0)$ where

$$
n(r-, 0)=\lim _{t \rightarrow r_{-}} n(t, 0) .
$$

REMARK 1 . Obviously $n(r, 0) \leqslant N$ and so (5) gives Bernstein's inequality. If none of the zeros of $Q$ lies in $|z|<r$, then $n(r-, 0)=0$ and (5) implies the Erdös-Lax inequality.

REMARK 2. In its present form, the conjecture is not true. A counterexample is given in $\$ 4$ below. A correct formulation of the conjecture requires that the polynomials $Q$ be suitably restricted.

In this note we shall prove the above conjecture for a class $\mathcal{H}$ of polynomials defined as follows: $\mathcal{H}$ is the set of all polynomials $Q$ such that:

(i) $Q$ has only real coefficients;

(ii) all the zeros of $Q$ lie in the half-plane $\operatorname{Re}(z) \leqslant 0$.

If $Q$ is a polynomial that satisfies (5), one easily verifies that each of the following polynomials also satisfies (5):

1. $c z^{m} Q(z)$, where $c$ is a constant and $m(\geqslant 0)$ is an integer;

2. $Q\left(e^{i \alpha} z\right)$, where $\alpha$ is a real number.

In particular, if (5) holds for all polynomials $Q$ in the class $\mathcal{H}$, then it also holds for polynomials all of whose zeros lie on one ray, as well as polynomials whose zeros are symmetrically placed along one line through the origin, e.g. the Legendre polynomials. This is because these later ones are obtained from some members of $\mathcal{H}$ by application of principles 1 and 2 . Thus, although (5) is not true in general, the class of polynomials for which the results of this note apply is large enough to include many of the interesting and well-known classes of polynomials.

2. Statement of results. Throughout this paper we retain the notation of the introduction.

THEOREM 1. Let $Q$ be a polynomial in the class $\mathcal{H}$ having degree $N(>0)$. Then

$$
M\left(r, Q^{\prime}\right) \leqslant \frac{N+n(r, 0)}{2 r} M(r, Q) \quad(r>0) .
$$

More precisely, (7) continues to hold if $n(r, 0)$ is replaced by $n(r-, 0)$.

It is easy to see that $\mathcal{H}$ is closed under differentiation. This fact, combined with Theorem 1, gives the following corollary, whose proof we shall omit.

Corollary 1. Let $Q \in \mathcal{H}$ be of degree $N(>0)$. Then

$$
M\left(r, Q^{(k)}\right) \leqslant\left\{\prod_{j=0}^{k-1}\left\{\frac{N-j+n\left(r, 0 ; Q^{(j)}\right)}{2 r}\right\}\right\} M(r, Q),
$$

where $Q^{(k)}$ is the kth derivatrive of $Q, Q^{(0)}=Q$ and $r>0$. In particular, if $a_{N}$ is the leading coefficient of $Q$, then

$$
N !\left|a_{N}\right| \leqslant\left\{\prod_{j=0}^{N-1}\left(\frac{N-j+n\left(r, 0 ; Q^{(j)}\right)}{2 r}\right)\right\} M(r, Q) .
$$


We note that since $n\left(r, 0 ; Q^{(j)}\right) \leqslant N-j$, the estimate on $a_{N}$ given by (9) is sharper than the well-known Cauchy estimate $\left|a_{N}\right| \leqslant r^{-N} M(r, Q)$.

Our next result is a converse to the Conjecture.

THEOREM 2. Let $Q$ be an entire function and let $n(r, 0)$ be the counting function of its zeros. Assume there exists a constant $\alpha(\geqslant 0)$ such that

$$
M\left(r, Q^{\prime}\right) \leqslant \frac{\alpha+n(r, 0)}{2 r} M(r, Q)
$$

for every $r>0$. Then $Q$ is a polynomial of degree $\leqslant \alpha$.

Since Bernstein's inequality holds for all polynomials, Theorem 2 suggests the following characterization of polynomials:

COROLlaRY 1. Let $Q$ be an entire function and let $\alpha(\geqslant 0)$ be a constant. Then $Q$ is a polynomial of degree at most $\alpha$ if and only if the inequality

$$
M\left(r, Q^{\prime}\right) \leqslant(\alpha / r) M(r, Q) \quad(r>0)
$$

holds.

3. Proof of Theorem 1. Let $Q \in \mathcal{K}$ be of degree $N(>0)$. By the discussion after Remark 2 in the Introduction, we may assume $Q(0)=1$. Since the coefficients of $Q$ are all real, its zeros will either be real negative or will appear in conjugate pairs in the left half-plane. Denote the real zeros of $Q$ by $\left\{-a_{\mu}\right\}_{\mu=1}^{m}$ where $0<a_{1} \leqslant a_{2} \leqslant$ $\cdots \leqslant a_{m}$. The nonreal zeros of $Q$ we denote by $\left\{z_{\nu}, \bar{z}_{\nu}\right\}_{\nu=1}^{p}$, where

$$
z_{\nu}=b_{\nu} e^{i\left(\pi-\beta_{\nu}\right)}, \quad 0<\beta_{\nu} \leqslant \pi / 2,
$$

and $0<b_{1} \leqslant b_{2} \leqslant \cdots \leqslant b_{p}$. If we put

$$
u_{\mu}(z)=\left(1+z / a_{\mu}\right)
$$

and

$$
v_{\nu}(z)=\left(1-z / z_{\nu}\right)\left(1-z / \bar{z}_{\nu}\right)=1+2 \cos \beta_{\nu}\left(z / b_{\nu}\right)+z^{2} / b_{\nu}^{2},
$$

then $Q(z)$ takes the form

$$
Q(z)=\prod_{\mu=1}^{m} u_{\mu}(z) \prod_{\nu=1}^{p} v_{\nu}(z) \quad(m+2 p=N) .
$$

The restriction on $\beta_{\nu}$ shows that $\cos \beta_{\nu} \geqslant 0$ and this, together with (12), implies

$$
Q(z)=\sum_{k=0}^{N} c_{k} z^{k}
$$

where $c_{k} \geqslant 0$ for all $0 \leqslant k \leqslant N$.

It follows that $M(r, Q)=Q(r)$ and $M\left(r, Q^{\prime}\right)=Q^{\prime}(r)$, therefore, by (12),

$$
\frac{M\left(r, Q^{\prime}\right)}{M(r, Q)}=\sum_{\mu=1}^{m} \frac{1}{r+a_{\mu}}+\sum_{\nu=1}^{p} \frac{v_{\nu}^{\prime}(r)}{v_{\nu}(r)} .
$$


Let $n_{\mu}(r)$ and $n_{\nu}(r)$ be the counting functions of $u_{\mu}(z)$ and $v_{\nu}(z)$, respectively. Then

$$
\begin{aligned}
n_{\mu}(r) & =0, \quad r<a_{\mu}, \\
& =1, \quad r \geqslant a_{\mu} \quad(1 \leqslant \mu \leqslant m),
\end{aligned}
$$

and

$$
\begin{aligned}
& n_{\nu}(r)=0, \quad r<b_{\nu}, \\
& =2, \quad r \geqslant b_{\nu} \quad(1 \leqslant \nu \leqslant p) .
\end{aligned}
$$

From (2) and (3) it readily follows that

$$
\begin{aligned}
& \frac{v_{\nu}^{\prime}(r)}{v_{\nu}(r)} \leqslant \frac{2+n_{\nu}(r)}{2 r} \text { and } \frac{1}{r+a_{\mu}} \leqslant \frac{1+n_{\mu}(r)}{2 r} \\
& (1 \leqslant \mu \leqslant m, 1 \leqslant \nu \leqslant p, r>0) .
\end{aligned}
$$

Using (16) in (13) we obtain

$$
\begin{aligned}
\frac{M\left(r, Q^{\prime}\right)}{M(r, Q)} & \leqslant \sum_{\mu=1}^{m} \frac{1+n_{\mu}(r)}{2 r}+\sum_{\nu=1}^{p} \frac{2+n_{\nu}(r)}{2 r} \\
& =\frac{m+2 p}{2 r}+\frac{1}{2 r}\left\{\sum_{\mu=1}^{m} n_{\mu}(r)+\sum_{\nu=1}^{p} n_{\nu}(r)\right\} .
\end{aligned}
$$

Now (7) follows from (17) since

$$
n(r, 0)=n(r, 0 ; Q)=\sum_{\mu=1}^{m} n\left(r, 0 ; u_{\mu}\right)+\sum_{\nu=1}^{p} n\left(r, 0 ; v_{\nu}\right) \text {. }
$$

If $Q$ has no zeros on $|z|=r$ then $n(r, 0)=n(r-, 0)$. If $Q$ has zeros on $|z|=r$, then it has no zeros on $|z|=r-\varepsilon$ for a sufficiently small $\varepsilon$. Replacing, in (7), $r$ by $r-\varepsilon$, letting $\varepsilon \rightarrow 0$ and using the continuity of $M(r, Q)$ we see that (7) holds with $n(r-, 0)$. This finishes the proof of Theorem 1 .

Proof of THEOREM 2. Let $Q$ be an entire function satisfying the hypothesis of Theorem 2. We may assume $Q(z)$ is not identically zero since, otherwise, there would be nothing to prove. Then the zeros of $Q$ are at most countable and $n(r, 0: Q)$ is, for each $r$, finite.

Let $z=r e^{i \theta}$ be a given complex number. Then

$$
\begin{aligned}
Q(z) & =Q(0)+\int_{0}^{r} Q^{\prime}\left(t e^{i \theta}\right) e^{i \theta} d t \\
& =Q(0)+\left(\int_{0}^{a}+\int_{a}^{r}\right)\left(Q^{\prime}\left(t e^{i \theta}\right) e^{i \theta} d t\right)
\end{aligned}
$$

where $a(>0)$ is a fixed real number.

From (18) and (10) we obtain, by adding if necessary a positive constant,

$$
M(r, Q) \leqslant c+\int_{a}^{r} \frac{\alpha+n(t, 0)}{2 t} M(t, Q) d t \quad(r>a)
$$

where $c$ is a positive constant. 
In each interval $[a, r]$, the function $M(t, Q)$ is well known to be continuous, while $n(t, 0)$ is continuous except possibly for a finite number of points there. Consequently the integral in (19) is an absolutely continuous function of $r$ whose derivative with respect to $r$ equals $(\alpha+n(r, 0)) M(r, Q) / 2 r$ for almost all $r$. Furthermore, since $c>0$, the function

$$
F(r)=\log \left\{c+\int_{0}^{r} \frac{\alpha+n(t, 0)}{2 t} M(t, Q) d t\right\}
$$

is also absolutely continuous on any interval $[a, x]$ where $\mathrm{a}>0$. In particular,

$$
F(x)-F(a)=\int_{a}^{x} F^{\prime}(t) d t
$$

Now in (19), divide both sides of the inequality by its (positive) right-hand side. Then multiply both sides of the resulting inequality by $(\alpha+n(r, 0)) / 2 r$. Integrating the result between $a$ and $x$, we obtain

$$
F(x)-F(a) \leqslant \int_{a}^{x} \frac{\alpha+n(r, 0)}{2 r} d r=\frac{\alpha}{2} \log \left(\frac{x}{a}\right)+\frac{1}{2} \int_{a}^{x} \frac{n(r)}{r} d r .
$$

It is a simple consequence of Jensen's formula [3, p. 125] that

$$
\int_{a}^{x} \frac{n(r, 0)}{r} d r \leqslant \log M(x, Q)+c_{1}
$$

where $c_{1}$ is a positive constant.

It follows that

$$
F(x)-F(a) \leqslant(\alpha / 2) \log (x / a)+\frac{1}{2} \log M(x, Q)+c_{1} .
$$

Combining (19), (20) and (21) we obtain

$$
\log M(x, Q) \leqslant F(a)+(\alpha / 2) \log (x / a)+\frac{1}{2} \log M(x, Q)+c_{1}
$$

which implies that

$$
M(x, Q) \leqslant c_{2}(x / a)^{\alpha} \quad(x \geqslant a)
$$

where $c_{2}$ is a positive constant.

Write

$$
Q(z)=\sum_{n=0}^{\infty} a_{n} z^{n}
$$

If $n>\alpha$ then Cauchy's inequality [3, p. 84] and (22) give

$$
n !\left|a_{n}\right| \leqslant r^{-n} M(r, Q) \leqslant c_{3} r^{-n+\alpha} \quad(r \geqslant a) .
$$

Letting $r \rightarrow \infty$ in (24) we conclude that $a_{n}=0$ for all $n>\alpha$. Hence $Q$ is a polynomial of degree $\leqslant \alpha$. This finishes the proof of Theorem 2 .

For the proof of Corollary 1 (of Theorem 2), one part is Bernstein's inequality. For the other part of the proof, let $Q$ be an entire function and suppose that (11) holds for some constant $\alpha(\geqslant 0)$. Then (10) holds with $\alpha$ replaced by $2 \alpha$, and so, by Theorem 2, the function $Q$ is a polynomial of degree $\leqslant 2 \alpha$. But then by writing

$$
Q(z)=c_{m} z^{m}+c_{m-1} z^{m-1}+\cdots+c_{0} \quad\left(c_{m} \neq 0\right),
$$


we see that for $r \rightarrow \infty$,

$$
M(r, Q) \sim\left|a_{m}\right| r^{m}, \quad M\left(r, Q^{\prime}\right) \sim m\left|a_{m}\right| r^{m-1}
$$

and, hence,

$$
\alpha \geqslant r M\left(r, Q^{\prime}\right) / M(r, Q) \sim m .
$$

Thus $Q$ is a polynomial of degree at most $\alpha$.

4. A counterexample. If the class $\mathcal{H}$ is replaced by the class $\mathscr{P}$ consisting of polynomials with only nonnegative coefficients, does the conclusion of Theorem 1 hold for polynomials $Q$ in $\mathscr{P}$ ?

The following example will show that the answer is negative. Let

$$
Q(z)=(z+1)\left(z^{4}+2\right)=z^{5}+z^{4}+2 z+2 .
$$

Then $Q \in \mathscr{P}$ and

$$
\frac{M\left(r, Q^{\prime}\right)}{M(r, Q)}=\frac{5 r^{4}+4 r^{3}+2}{r^{5}+r^{4}+2 r+2} .
$$

If $Q$ satisfied the conclusion of Theorem 1 , then for $r=\sqrt{2}$, we would have

$$
\frac{M\left(r, Q^{\prime}\right)}{M(r, Q)} \leqslant \frac{5+1}{2 r}=\frac{3}{r} \quad(r=\sqrt{2})
$$

or

$$
f(r)=2 r^{5}+r^{4}-4 r-6 \leqslant 0 \text { for } r=\sqrt{2},
$$

which is false for all $r$ in a sufficiently small neighbourhood of $\sqrt{2}$ since $f$ is continuous and $f(\sqrt{2})=4 \sqrt{2}-2>0$.

We end this note by the following:

QUESTION. What is the analogue of Theorem 1 for polynomials all of whose roots lie in the left half-plane $\operatorname{Re} z \leqslant 0$ ?

\section{REFERENCES}

1. Abdul Aziz and Q. G. Mohammad, Simple proof of a theorem of Erdös and Lax, Proc. Amer. Math. Soc. 80 (1980), 119-122.

2. J. E. Littlewood, Lectures on the theory of functions, Oxford Univ. Press, 1944.

3. E. C. Titchmarsh, The theory of functions (2nd ed.), Oxford Univ. Press, 1939.

Department of Mathematics, American University of Beirut, Beirut, Lebanon 